\title{
Parton Distributions for LHC
}

\author{
S. Alekhin ${ }^{\mathrm{a} *}$

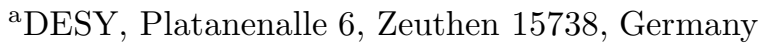

I review the current status of the nucleon PDFs determined from global fits with emphasis on the impact of recent experimental data and the remaining theoretical challenges.

The detailed knowledge of the momentum distributions of partons in the nucleon is quite necessary for phenomenology of the hard scattering processes. Since the time, when the parton model was established the parton distribution functions (PDFs) were permanently in the focus of the QCD studies. With a tremendous upgrade of the Tevatron collider luminosity and the start-up of the LHC at a record collision energy we need, however, an even better understanding of the PDF details in order to meet the improving accuracy of the experimental data. The PDFs are extracted from the hard-scattering data with the QCD evolution taken into account. At the present accuracy of the experimental data, moreover for the foreseen precision at the LHC, the QCD corrections at least up to the next-toleading order (NLO) are required. Furthermore, the next-to-next-to-leading order (NNLO) corrections are necessary for many important processes, as $W, Z$-bosons, Higgs boson and top-quark production.

The choice of the data used to constrain the PDFs is often defined by the accuracy of the available theoretical calculations. For example, in order to include the DIS data with low momentum transfers $Q^{2}$ into the PDF fit, one has to take into account the higher-order QCD corrections and to take care about the higher twist terms for the small-mass final state kinematics. Likewise, modifications of the nucleon wave function in nuclei have to be taken into account once nuclear data are used in the analysis.

\footnotetext{
*Permanent address: Institute for High Energy Physics, Pobeda 1, Protvino 142281 Russia
}

There are several nucleon PDF sets, which were kept updated during the recent years, cf. Table 1. All these PDF sets are based on the inclusive deep-inelastic-scattering (DIS) data collected in fixed-target experiments and the data from the HERA collider. The HERA data mostly provide constraints on the gluon and sea quarks distributions at small values of the Bjorken variable $x$, which are particularly important for the interpretation of the future data of the first LHC run. Recently the inclusive data obtained by the $\mathrm{H} 1$ and ZEUS experiments of Run I of the HERA collider were combined into the common data set [6]. The advantage of such an approach is mutual crosscalibration of both experiments, which leads to an essential improvement in the data accuracy. The slope of the updated HERA data on the inclusive structure function (SF) $F_{2}$ with respect to $\ln \left(Q^{2}\right)$, obtained from a model-independent fit, is given in Fig. 1. It is in good agreement with the NNLO predictions based on the ABKM09 PDFs [7]. However, the constant term obtained in the same fit systematically overshoots the predictions. The combined HERA data in general lie above the separate data sets of Ref. [8] used in the PDF fits before. This change in the data requires a modification of the small- $x$ shape of the PDFs. In particular, for the version of the ABKM09 fit based on the combined HERA data, the gluon distribution at small $x$ is shifted to lower values, cf. Fig. 2. In the updated version of the NLO fit by the CTEQ collaboration a decrease of the gluon distribution at small $x$ is also observed [2]. However, the MSTW group found much smaller variations of the PDFs [10].

The PDF sets of Refs. [1-3] were fitted also 
Table 1

Recently published nucleon PDFs sets with a brief description of the data used in the fit, the theoretical accuracy and the factorization scheme employed to model the heavy-quark DIS contribution.

\begin{tabular}{lllll}
\hline Name & Data used & QCD approximation & Scheme & Reference \\
\hline MSTW & DIS+DY+jets & NLO/NNLO & GMVFN & {$[1]$} \\
CTEQ & DIS+DY+jets & NLO & GMVFN & {$[2]$} \\
NNPDF & DIS+DY+jets & NLO & ZMVFN & {$[3]$} \\
JR & DIS+DY & NLO/NNLO & FFN & {$[4]$} \\
ABKM & DIS+DY & NLO/NNLO & FFN & {$[5]$} \\
HERAPDF & DIS & NLO & GMVFN & {$[6]$} \\
\hline
\end{tabular}

to the data on the $W^{ \pm}$and $Z$ production from hadron colliders. These collider data provide important constraints on the PDFs at large values of the factorization scale. The $W^{ \pm}, Z$ rates and distributions are calculated at NNLO $[11,12]$ and are considered as one of the LHC luminosity monitors. However, the most recent and accurate Run II Tevatron data [13] on the $W$ charge asymmetry and the related data on the lepton charge asymmetry are not in a good agreement with the NNLO calculations of Ref. [12] based on the different PDFs, particularly for the case of the electron charge asymmetry data. The best agreement was observed for the case of the JR PDFs of Ref. [4], cf. Fig. 3. The MSTW and ABKM09 predictions overshoot the data in general. The NNPDF collaboration also obtained a poor description of the $W$-asymmetry data in their NLO fit [3]. Due to the QCD evolution of the PDFs the kinematics of the $W$-production at the Tevatron is constrained by the fixed target DIS data at $x=0.1 \div 0.2$. Therefore the $W$-asymmetry predictions are mostly sensitive to the valence quark isospin asymmetry, $(u-d)$. This distribution can be constrained by the data on isospin-asymmetric combination of the proton and the neutron DIS SFs. However, the neutron target is available in a form of a bound state only. Therefore the neutron SFs unfolding requires to take into account nuclear effect. The DIS nuclear corrections are non-negligible even for the lightest nuclei, as the deuteron. Furthermore, they cannot be fully calculated using present theoretical methods in nuclear physics and are often fitted to nuclear data instead, cf. e.g. Ref [14]. The MSTW group included into their fit the deuteron correction in a form of a model-independent function and fitted it to the global data set [10]. In this way they found marginal improvement for the charged lepton asymmetry. Moreover, a reasonable nuclear model cannot justify the form of the deuteron correction obtained. Besides, the data on the lepton asymmetry obtained by two Fermilab experiments, CDF and D0, are not in agreement. In view of these problems the CTEQ group suggests two variants of their recent $P D F$ release, with and without Run II Tevatron data included [2].

The Tevatron jet inclusive production data were also used in Refs. [1-3]. Historically, these data were always considered as a unique source of the information about the gluon distribution at large $x$. Since the NNLO corrections to the hadronic jet production are not yet known these data can be used in a fully consistent way for extraction of the NLO PDFs only. With the Run I data of Ref. [15] included into the PDF fit, the CTEQ and MSTW groups observed a substantial increase of the large- $x$ gluons $[16,17]$. In fact, this effect was a manifestation of the tension between the Run I jet data and the DIS data at large $x$. The accuracy of the data obtained in the Run II experiments has been greatly improved, in particular due to the better jet energy calibration. Furthermore, these data lie lower than those from Run I and are in much better agreement with the other data used in the PDF fits. As a result, the impact of the Run II results on the PDFs is only minor, cf. Fig. 4. Due to this change of trend, 


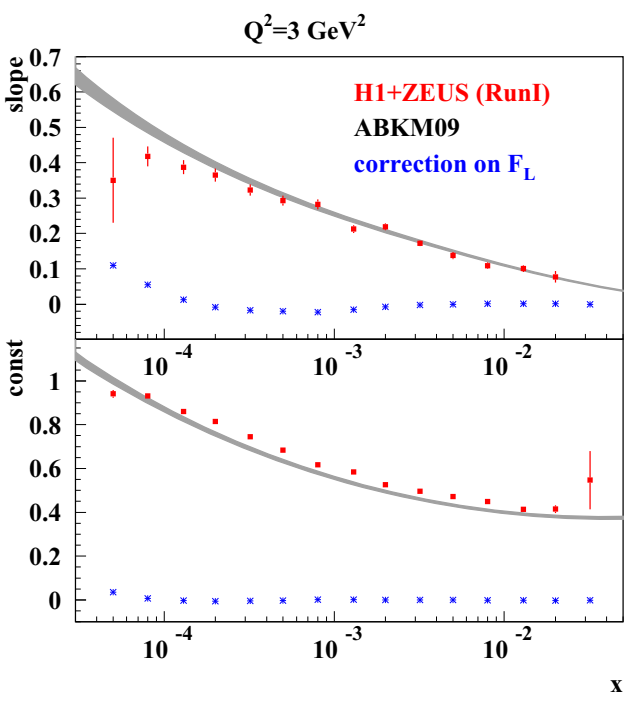

Figure 1. The slope $d F_{2} / d \ln Q^{2}$ (upper panel) and the constant term (lower panel) for the combined HERA Run I data on the inclusive SF $F_{2}$ in comparison to the predictions of the ABKM09 fit [5]. The impact of the correction on the contribution of the longitudinal SF $F_{\mathrm{L}}$, which were employed to extract the values of $F_{2}$ from the cross sections data is given by stars. Figure taken from Ref. [7].

the value of the strong coupling constant $\alpha_{\mathrm{s}}$ extracted from the Tevatron data is shifted downward. The shift in the value of $\alpha_{\mathrm{s}}$ is essential since in leading order QCD the jet production cross section is $O\left(\alpha_{\mathrm{s}}^{2}\right)$. The value of $\alpha_{\mathrm{s}}\left(M_{Z}\right)=$ $0.1161_{-0.0018}^{+0.0011}$ was obtained in the recent analysis of the D0 data performed at the NLO with account of NNLO threshold corrections [18]. Taking into account that the $\alpha_{\mathrm{s}}^{N N L O}$ is smaller than $\alpha_{\mathrm{s}}^{N L O}$, this result is in a good agreement with the value of $\alpha_{\mathrm{s}}^{N N L O}\left(M_{Z}\right)=0.1147(12)$ obtained in the updated version of the ABKM fit [7] and with $\alpha_{\mathrm{s}}^{N 3 L O}\left(M_{Z}\right)=0.1141_{-0.0022}^{+0.0020}$ obtained in the analysis of the non-singlet DIS data [19]. The value of $\alpha_{\mathrm{s}}^{N L O}\left(M_{Z}\right)=0.1215$ obtained in the updated version of the MSTW fit [10] is much larger than both these determinations. For comparison,

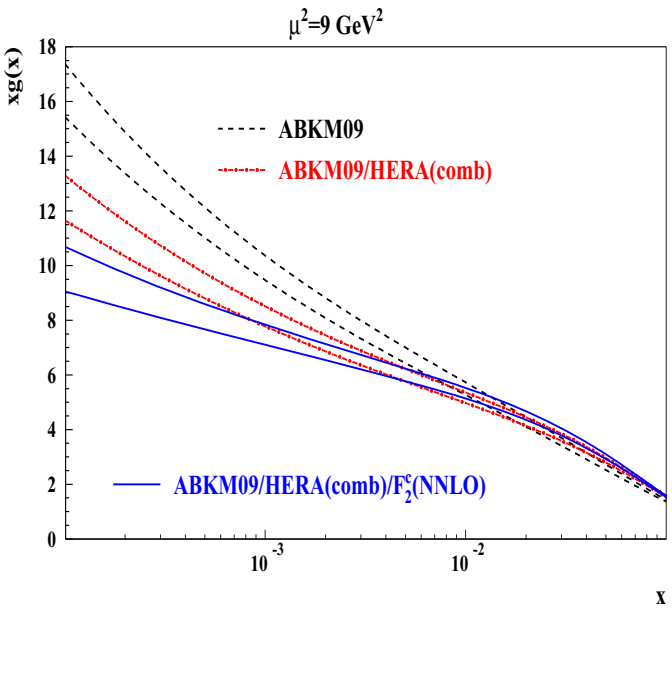

Figure 2. The $1 \sigma$ bands for the NNLO gluon distribution obtained in the updated version of our fit (blue) compared to the same for ABKM09 fit (black) and the version of the updated fit without partial NNLO corrections to the heavy-quark electro-production of Ref. [9] taken into account (red) at the factorization scale of $9 \mathrm{GeV}^{2}$. Figure taken from Ref. [7].

the value of $\alpha_{\mathrm{s}}$ extracted from the $e^{+} e^{-}$data is $\sim 0.119$ [20]. However, it is worth to note that in the elaborated analysis of the global $e^{+} e^{-}$data on the thrust distribution performed in the NNLO approximation with account of the power corrections to fragmentation the value of $\alpha_{\mathrm{s}}\left(M_{Z}\right)=$ $0.1135 \pm(0.0002)_{\text {exp. }} \pm(0.0005)_{\text {hadr. }} \pm(0.0009)_{\text {pert. }}$. was obtained [21]. This is much lower than the average of Ref. [20] and in good agreement with the determinations based on the DIS data.

The H1 and ZEUS data on the DIS charm production obtained in Run I of the HERA collider have also been combined into a common data set [22] recently. Heavy-quark electro-production is particularly sensitive to the shape of the gluon distribution at small $x$. Therefore it gives a complimentary constraint for the global PDF fits. Moreover, the detailed understanding of the heavy-quark DIS electro-production mechanism is necessary for the small- $x$ inclusive SF de- 


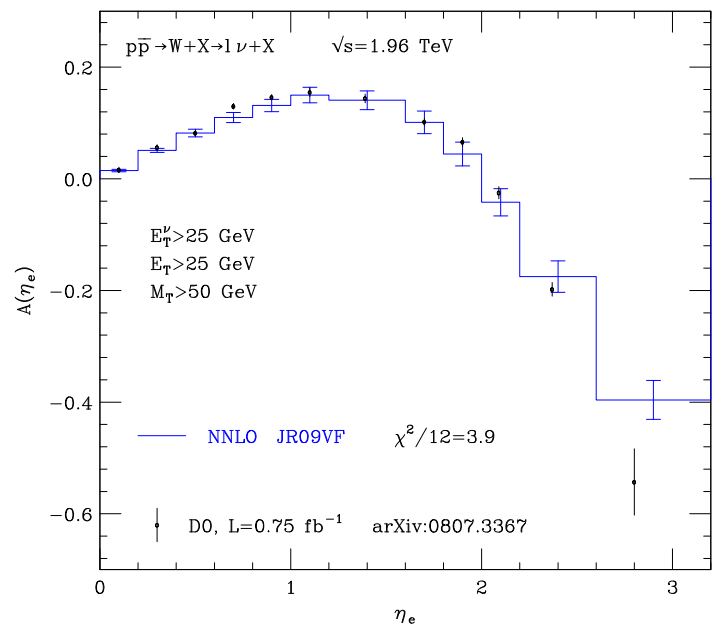

Figure 3. The Run II electron charge asymmetry obtained by the D0 collaboration in comparison with the NNLO predictions based on the JR PDFs [4]. Figure taken from Ref. [12].

scription. In the global PDF fits the zero-mass variable-flavor-number (ZMVFN) scheme is often used to model the heavy-quark contribution. In this scheme all quarks are assumed to be massless, which greatly simplifies the calculation. However, the low- $Q^{2}$ part of the DIS SFs cannot be calculated within this concept due to the power corrections and different constant terms occurring in the coefficient functions. The ZMVFN scheme commonly overestimates the heavy-quark contribution at small $Q^{2}$. Therefore it cannot be employed for the analysis of realistic DIS data. Instead of this the general-mass variable-flavornumber (GMVFN) scheme is used. The GMVFN schemes include a peculiar modeling of the low$Q^{2}$ DIS region. This allows to overcome problems of the ZMVFN scheme. However, since the modeling cannot be performed on solid theoretical grounds the GMVFN scheme cannot be defined in a unique way. Various variants of the GMVFN scheme were considered, cf. Ref. [23] and references therein. Further modifications of the ACOT scheme [24] and the updated version of Thorne's prescription [25] were suggested. The

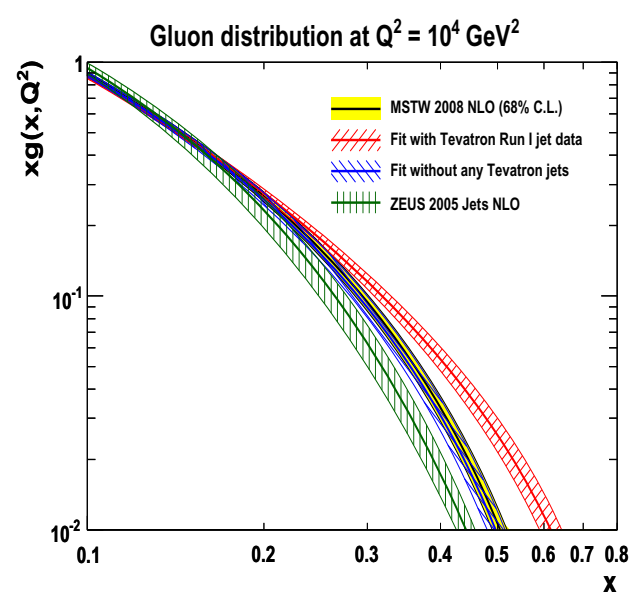

Figure 4. The large- $x$ gluon distribution obtained in different variants of the NLO MSTW08 fit. Figure taken from Ref. [1].

latter assumes a very flexible form of the semiinclusive heavy-quark $\mathrm{SF} F_{2}^{h}$ at low $Q^{2}$, which is provided by several additional parameters. An additional flexibility of model [25] would evidently allow better description of the data within this approach. However, additional parameters introduced in the GMVFN schemes to model the DIS SFs at low $Q^{2}$ have to keep the factorization scheme consistency. Otherwise the PDFs obtained in the fit based on the GMVFN scheme might correspond to some peculiar factorization scheme, but not to the $\overline{\mathrm{MS}}$ scheme, commonly used in the QCD calculations, cf. Ref. [26]. In contrast, the BMSN prescription of the GMVFN scheme [27] gives a consistent $\overline{\mathrm{MS}}$ description of the heavy-quark contribution in the transition region. At the same time it provides a smooth transition between the FFN scheme at low $Q^{2}$ and the ZMVFN scheme at large $Q^{2}$, cf. Fig. 5. These advantages of the BMSN ansatz were also employed in the FONLL approach to DIS considered in Ref. [28].

In the fixed-flavor-number (FFN) scheme the heavy-quark mass dependence follows from a complete fixed-order computation without any 


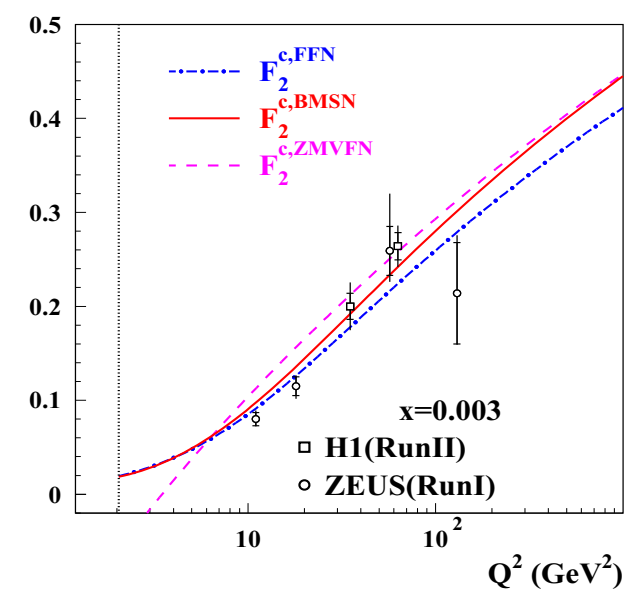

Figure 5. Comparison of $F_{2}^{c}$ in different schemes to H1- and ZEUS-data. Solid line: GMVFN scheme in the BMSN prescription, dash-dotted line: FFN scheme, dash line: ZMVFN scheme. Figure taken from Ref. [5].

modeling even at low $Q^{2}$. The coefficient functions of the heavy-quark semi-inclusive SFs are calculated in complete form up to the $O\left(\alpha_{\mathrm{s}}^{2}\right)$ (NLO) [29] semianalytically. At the values of $Q^{2} \gg m_{h}^{2}$ the fixed-order FFN expressions are insufficient since they do not include the big$\log$ terms of $O\left(\alpha_{\mathrm{s}}^{n} \ln ^{m}\left(Q^{2}\right)\right)$, which might be important even for large $n$. In the ZMVFN scheme these terms are resummed in a natural way through the QCD evolution of the heavyquark PDFs. This is often considered as an advantage of the ZMVFN schemes as compared to the FFN one. Nonetheless, these terms are partially taken into account in the NLO expressions of Ref. [29] and the numerical effect of the remaining big-log terms is marginal for the realistic data kinematics. This observation was first made in Ref. [30] and later confirmed in Ref. [5] for the case of recent data on $F_{2}^{c}$ and $F_{2}^{b}$ accumulated by the HERA experiments. In particular, the difference between the PDFs obtained in the FFN fit and in the GMVFN fit based on the BMSN

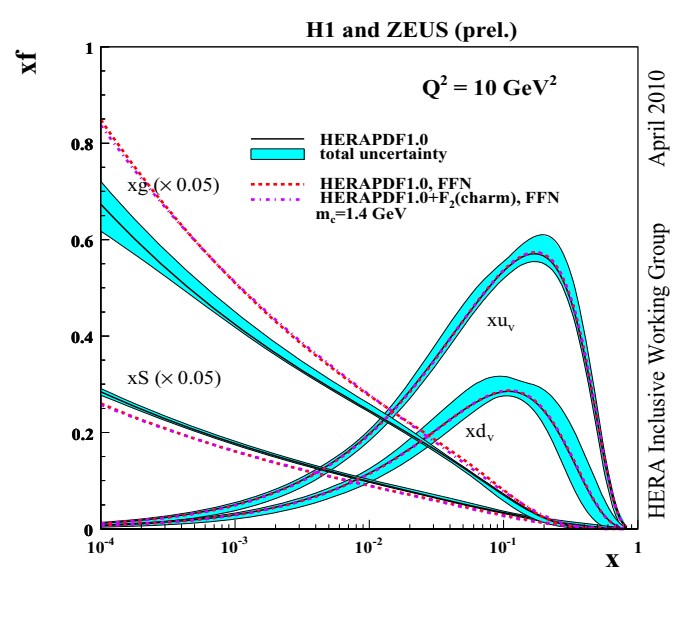

Figure 6. Comparison of PDFs obtained in Ref. [6] for the GMVFN scheme of Ref. [25] and in the variant of this fit for the FFN scheme. Figure taken from Ref. [22].

prescription was found to be marginal [5]. For the PDF fit, which includes the combined HERA data on $F_{2}^{c}$, the FFN scheme and the different variants of the GMVFN schemes provide equally good description of the data, cf. Ref. [22]. At the same time the gluon distributions obtained for the GMVFN scheme of Ref. [25] and the FFN PDFs are quite different, particularly for the small- $x$ gluons, cf. Fig. 6 . The ZMVFN gluons at small $x$ must take lower values than for the FFN scheme by the scheme definition. However, this does not fully explain the effect given in Fig. 6. Meanwhile this effect might be related to the discrepancy in the gluon distributions obtained in the fits of Ref. [1] and Ref. [5].

The gluon-gluon collision is the dominant mechanism for the production of Higgs bosons with masses $100 \lesssim M_{\mathrm{H}} \lesssim 300 \mathrm{GeV}$ in hadronhadron collisions. Furthermore, the leading order gluon-gluon term is $O\left(\alpha_{\mathrm{s}}^{2}\right)$. This makes the Higgs production rate very sensitive to the variation of the gluon shape and the value of $\alpha_{\mathrm{s}}$. The Higgs production rates at the Tevatron collider and at LHC energies calculated with account 


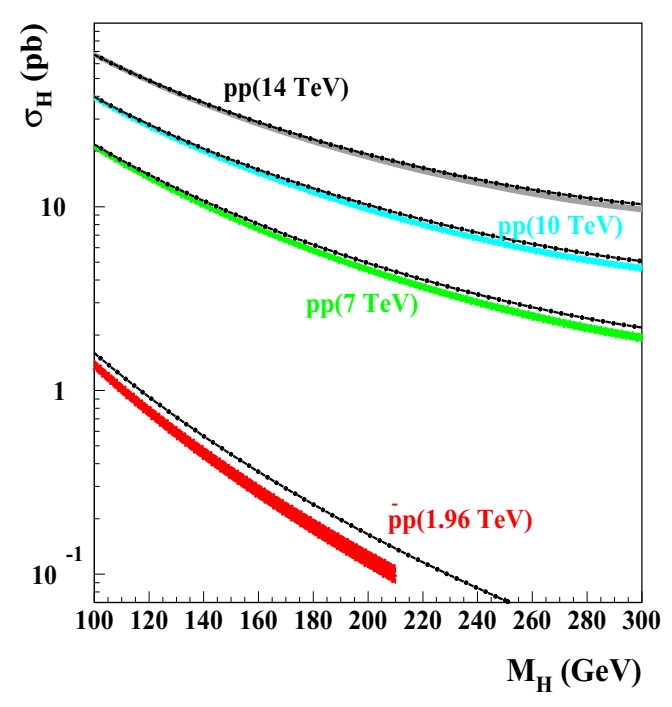

Figure 7. The $1 \sigma$ error band for the Higgs-boson production cross sections at Tevatron and the LHC employing the ABKM PDFs [5] in comparison with the central values for the case of MSTW PDFs [1] (dash-dotted lines). Figure taken from Ref. [5].

of the NNLO corrections of Ref. [31] are given in Fig. 7. The estimates based on the NNLO ABKM09 PDFs take somewhat lower values than those based on the MSTW08 PDFs, particularly for the case of Tevatron. This is explained by a cumulative effect of the difference in the gluon distribution and $\alpha_{\mathrm{s}}$ for these two sets. This discrepancy reduces the statistical significance of the constraint on the Higgs mass obtained at the Tevatron collider, cf. Refs. $[32,33]$. For the conclusive interpretation of the upcoming LHC results it would be useful to reduce the spread of the predictions.

In summary, the ensemble of nucleon PDF sets is maintained at the moment and is ready for phenomenological studies at the LHC. The PDFs are tuned to a variety of the hard-scattering data, including the most recent ones, and provide an accuracy of the LHC standard candle processes at the level of several percent [34]. Nonetheless the predictions based on the different PDFs are not in perfect agreement, presumably due to the distinct details in the theoretical treatment of the data employed by different groups. Clarification of these aspects and consolidation of the predictions will essentially improve the precision of the future LHC findings.

Acknowledgments. I would like to thank J. Blümlein for careful reading of the manuscript and valuable comments. This work was supported in part by Helmholtz Alliance "Physics at the Terascale".

\section{REFERENCES}

1. A. D. Martin, W. J. Stirling, R. S. Thorne and G. Watt, Eur. Phys. J. C 63 (2009) 189.

2. H. L. Lai, M. Guzzi, J. Huston, Z. Li, P. M. Nadolsky, J. Pumplin and C. P. Yuan, arXiv:1007.2241 [hep-ph].

3. R. D. Ball, L. Del Debbio, S. Forte, A. Guffanti, J. I. Latorre, J. Rojo and M. Ubiali, Nucl. Phys. B 838 (2010) 136.

4. P. Jimenez-Delgado and E. Reya, Phys. Rev. D 79, 074023 (2009).

5. S. Alekhin, J. Blümlein, S. Klein and S. Moch, Phys. Rev. D 81 (2010) 014032.

6. F. D. Aaron et al. [H1 Collaboration and ZEUS Collaboration], JHEP 1001, 109 (2010).

7. S. Alekhin, J. Blümlein and S. Moch, arXiv:1007.3657 [hep-ph].

8. C. Adloff et al. [H1 Collaboration], Eur. Phys. J. C 21 (2001) 33; S. Chekanov et al. [ZEUS Collaboration], Eur. Phys. J. C 21, 443 (2001).

9. S. Alekhin and S. Moch, Phys. Lett. B 672 (2009) 166.

10. R. S. Thorne, A. D. Martin, W. J. Stirling and G. Watt, arXiv: 1006.2753 [hep-ph].

11. C. Anastasiou, L. Dixon, K. Melnikov and F. Petriello, Phys. Rev. Lett. 91 (2003), 182002; Phys. Rev. D69, 094008 (2004).

12. S. Catani, G. Ferrera and M. Grazzini, JHEP 1005 (2010) 006.

13. D. E. Acosta et al. [CDF Collaboration], Phys. Rev. D 71 (2005) 051104; 
V. M. Abazov et al. [D0 Collaboration], Phys. Rev. D 77 (2008) 011106;

V. M. Abazov et al. [D0 Collaboration], Phys. Rev. Lett. 101 (2008) 211801.

14. S. A. Kulagin and R. Petti, Nucl. Phys. A 765 (2006) 126.

15. F. Abe et al. [CDF Collaboration], Phys. Rev. Lett. 77 (1996) 438;

B. Abbott et al. [D0 Collaboration], Phys. Rev. Lett. 86, 1707 (2001).

16. A. D. Martin, R. G. Roberts, W. J. Stirling and R. S. Thorne, Eur. Phys. J. C 23, 73 (2002).

17. H. L. Lai et al., Phys. Rev. D 55, 1280 (1997).

18. V. M. Abazov et al. [D0 Collaboration], Phys. Rev. D 80 (2009) 111107.

19. J. Blümlein, H. Böttcher and A. Guffanti, Nucl. Phys. B 774 (2007) 182.

20. S. Bethke, Eur. Phys. J. C 64 (2009) 689.

21. R. Abbate, M. Fickinger, A. H. Hoang, V. Mateu and I. W. Stewart, arXiv:1006.3080 [hep-ph].

22. A. M. Cooper-Sarkar, arXiv:1006.4471 [hep$\mathrm{ph}$.

23. R. S. Thorne and W. K. Tung, arXiv: 0809.0714 [hep-ph].

24. P. M. Nadolsky and W. K. Tung, Phys. Rev. D 79 (2009) 113014.

25. R. S. Thorne, arXiv:1006.5925 [hep-ph].

26. A. Chuvakin, J. Smith and W. L. van Neerven, Phys. Rev. D 61, 096004 (2000).

27. M. Buza, Y. Matiounine, J. Smith and W. L. van Neerven, Eur. Phys. J. C 1 (1998) 301;

I. Bierenbaum, J. Blümlein and S. Klein, Phys. Lett. B 672 (2009) 401.

28. S. Forte, E. Laenen, P. Nason and J. Rojo, Nucl. Phys. B 834, 116 (2010).

29. E. Laenen, J. Smith and W. L. van Neerven, Nucl. Phys. B 369, 543 (1992).

30. M. Glück, E. Reya and M. Stratmann, Nucl. Phys. B 422, 37 (1994).

31. R. V. Harlander and W. B. Kilgore, Phys. Rev. Lett. 88 (2002) 201801.

32. T. Aaltonen et al. [CDF and D0 Collaborations], Phys. Rev. Lett. 104 (2010) 061802.

33. J. Baglio and A. Djouadi, arXiv:1003.4266 [hep-ph].
34. The PDF4LHC Working Group Interim Report, to appear. 